\title{
A solution to the slow stabilisation of surface pressure sensors based on the Wilhelmy method
}

\author{
$\nabla$ Correspondence \\ s.w.james@cranfield.ac.uk \\ 8 Disciplines \\ Langmuir \\ Q Keywords \\ Monolayers \\ Wilhelmy \\ Langmuir \\ Surface Pressure \\ Langmuir Blodgett \\ it- Type of Observation \\ Standalone \\ O Type of Link \\ Standard Data
}

(O) Submitted Nov 11th, 2016 - Published Jan 12th, 2017

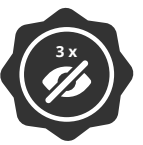

Triple Blind Peer Review The handling editor, the reviewers, and the authors are all blinded during the review process.

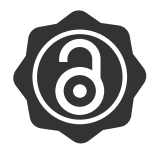

Full Open Access Supported by the Velux Foundation, the University of Zurich, and the EPFL School of Life Sciences.

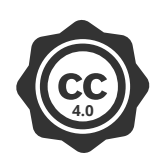

Creative Commons 4.0 This observation is distributed under the terms of the Creative Commons Attribution 4.0 International License.

\author{
Matthew Partridge, Frank Davis, Stephen W James, Seamus P Higson, Ralph P Tatam \\ Centre of Engineering Photonics, Cranfield University (GB); Department of Engineering and Applied Design, University of \\ Chichester (GB)
}

Dynamic measurement of surface pressure is of particular interest in the field of Langmuir monolayers, where the change in surface pressure throughout an experiment can provide information on the properties of the monolayer forming material, or on the reaction kinetics of the monolayer's interaction with other materials. One of the most common methods for the measurement of dynamic surface pressure is the Wilhelmy plate method. This method measures changes in the forces acting upon a thin plate of material at the air-water interface; this measurement is then converted to surface pressure. One version of this method, which uses filter paper plates at the air-water interface, is particularly popular due to their relatively low cost. However, it has been seen that the use of filter paper plates attached to a Wilhelmy balance requires an initial stabilisation period lasting several hours, during which the readings drift from the original baseline. Here the cause of this drift is explored, considering how changes in the weight of the plate over time influence the assumptions on which the surface pressure is derived from the measurements made by the Wilhelmy balance. A simple method for preventing this drift through pre-soaking of the filter paper plates is presented.

\section{Objective}

The cause of the drift in surface tension is not clear, but it is often attributed to contamination of the water in the trough, or to stabilisation of the response of the Wilhelmy plate and balance. In this paper a series of investigations aimed at developing an understanding of the origin of the drift, quantifying the problem and providing a solution are presented.

\section{Introduction}

The Wilhelmy method has been the mainstay of dynamic surface pressure measurement since its invention in the 1800 s by Ludwig Wilhelmy (Wilhelmy $\left.1863^{[1]}\right)$ The Wilhelmy method measures directly the change in the force acting upon a plate held at the interface between a liquid surface and the surrounding environment. When the plate is held at this interface, there is a combination of forces acting upon the plate; gravity, which pulls the plate into the liquid surface, the natural buoyancy of the plate in the liquid and the surface tension of the liquid, which pulls the plate into the water(Petty 1983 ${ }^{[2]}$ ) (Figure a). Dynamic measurement of the surface pressure is of particular interest in the field of Langmuir monolayers, where the change in surface pressure throughout an experiment can provide information on the properties of the monolayer forming materia)(Author B. $\left.2015^{[3]}\right)$ or on the reaction kinetics of the monolayer's interaction with other materials $(\mathrm{Su}-$ pian $\left.2010^{[4]}\right)$

The Wilhelmy plate measures changes in surface pressure through the change in the upward force exerted upon it by the liquid. As the surface tension decreases, the force acting on the plate, and thus weight of the plate, is reduced. The force acting on the plate is given by equation 1 .

(1) $F=$ Density $_{\text {plate }} g l w t+2 \gamma(t+w) \cos \theta+$ Density $_{\text {liquid }} g t w d$

Where the plate is defined by its thickness ( $\mathrm{t}$ ), width (w), length (l) and depth (d). The surface tension $(\mathrm{g})$ can be determined by rearranging equation 1 and assuming that the forces of gravity $(\mathrm{g})$ and buoyancy acting upon the plate remain constant throughout the experiment and that the contact angle (q) between the water and plate is zero (i.e. the plate is fully wet), 
(2) $\Delta \gamma=\frac{\Delta F(t+w)}{2}$

A Wilhelmy plate comprises either a piece of filter paper or a small plate of non-reactive material (often platinum). Platinum plates, while non-reactive to most monolayer materials, are difficult to clean and often require sonication and other treatments. This is partially due to the roughened surface of the platinum plates, which is required to encourage a zero degree contact angle between the plate and the liquid(Lapham 1999 ${ }^{[5]}$ ) Many laboratories opt for the cheaper, disposable alternative of a filter paper plate. The filter paper is naturally absorbent and so forms a reliable zero contact angle, though this absorbency can also lead to a 'wicking' effect causing the monolayer material to move up into the pad over time.

The use of filter paper Wilhelmy plates introduces another, less frequently reported, problem, which until now has not been understood fully. At the start of an experiment, the Wilhelmy plate is mounted on the balance with the plate immersed in the liquid. The plate is commonly left in this state for several hours while the system stabilises. During this stabilisation period, the readings recorded by the plate may drift downwards until a plateau is reached.

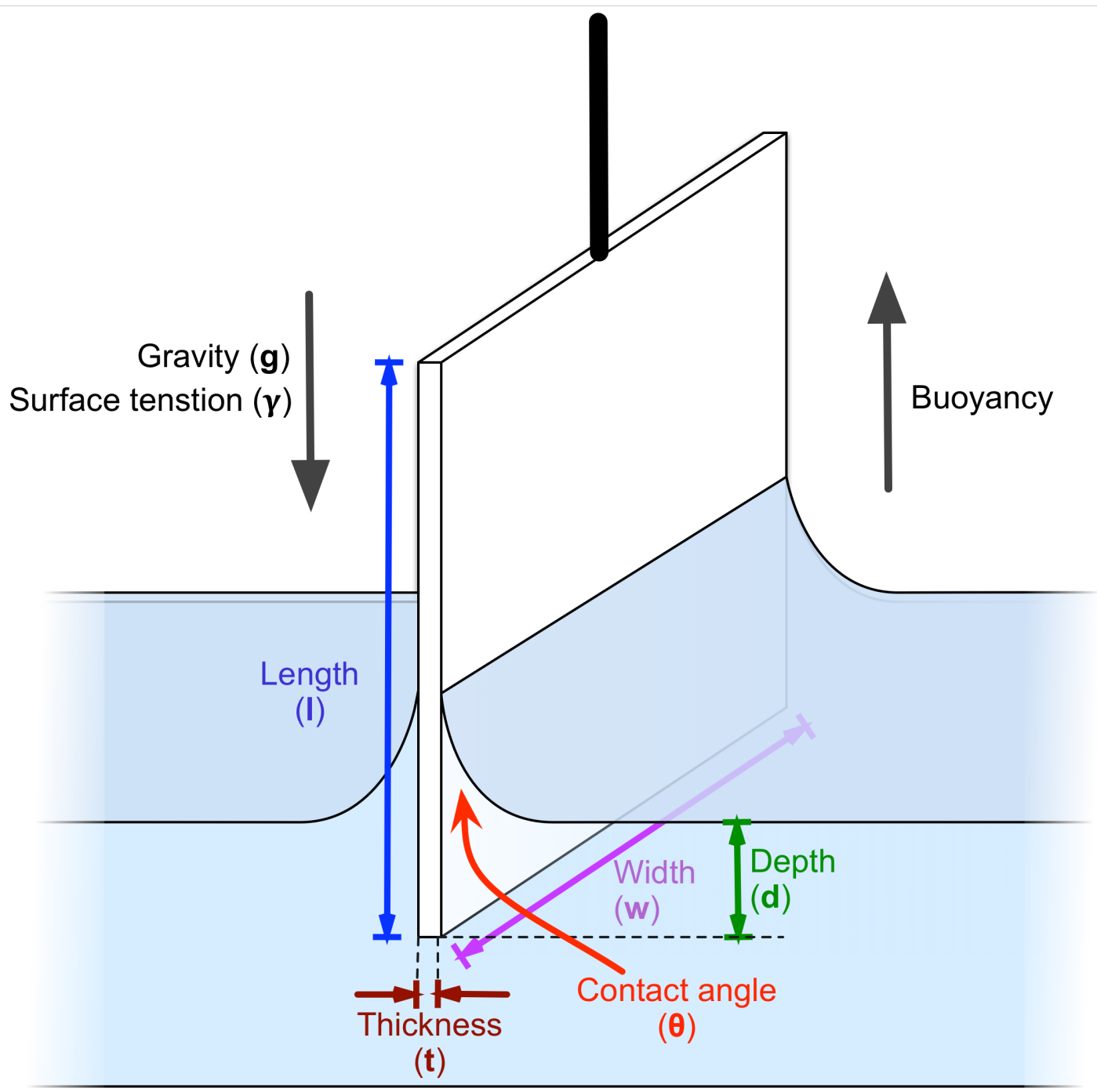


Time (minutes)

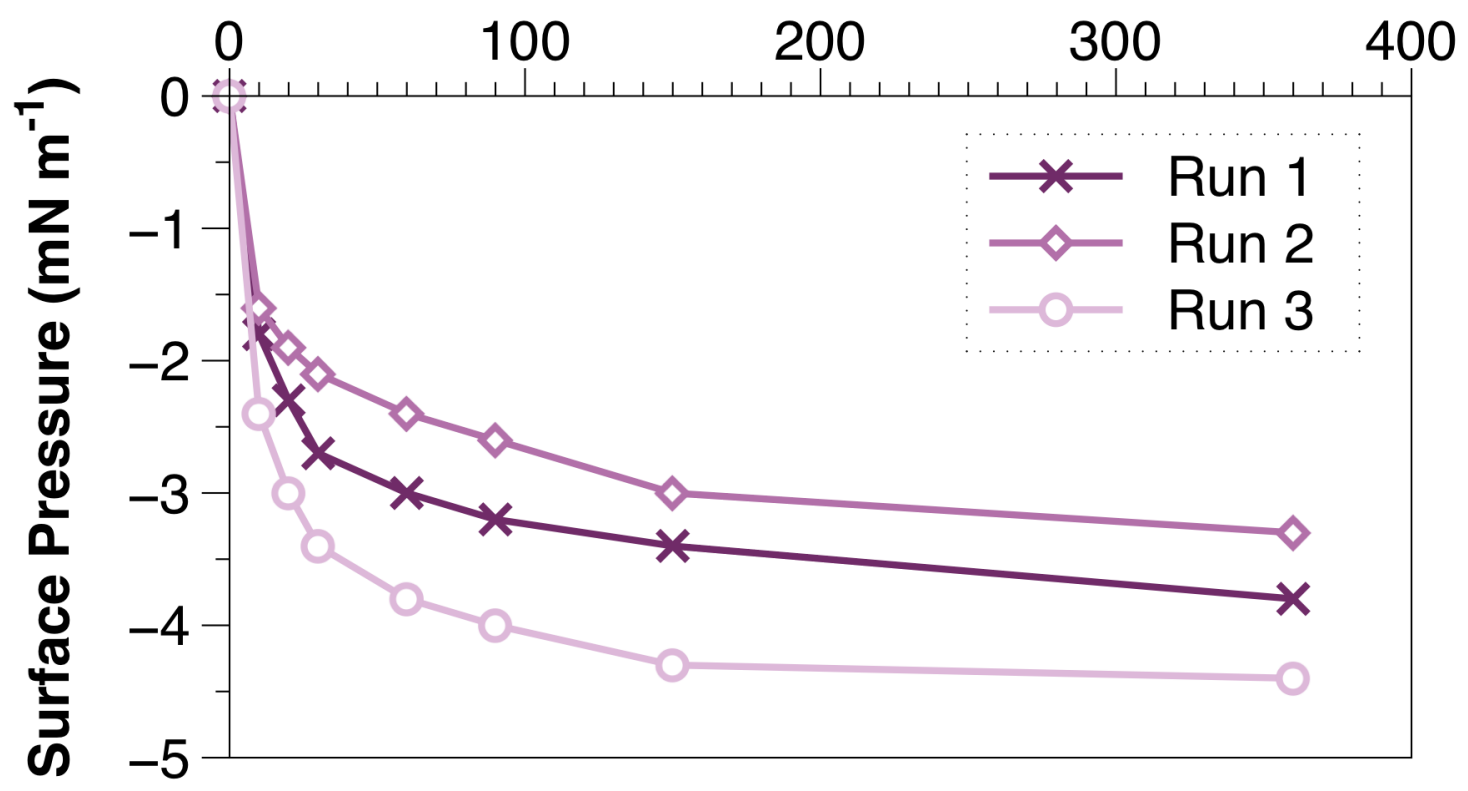

Time (minutes)

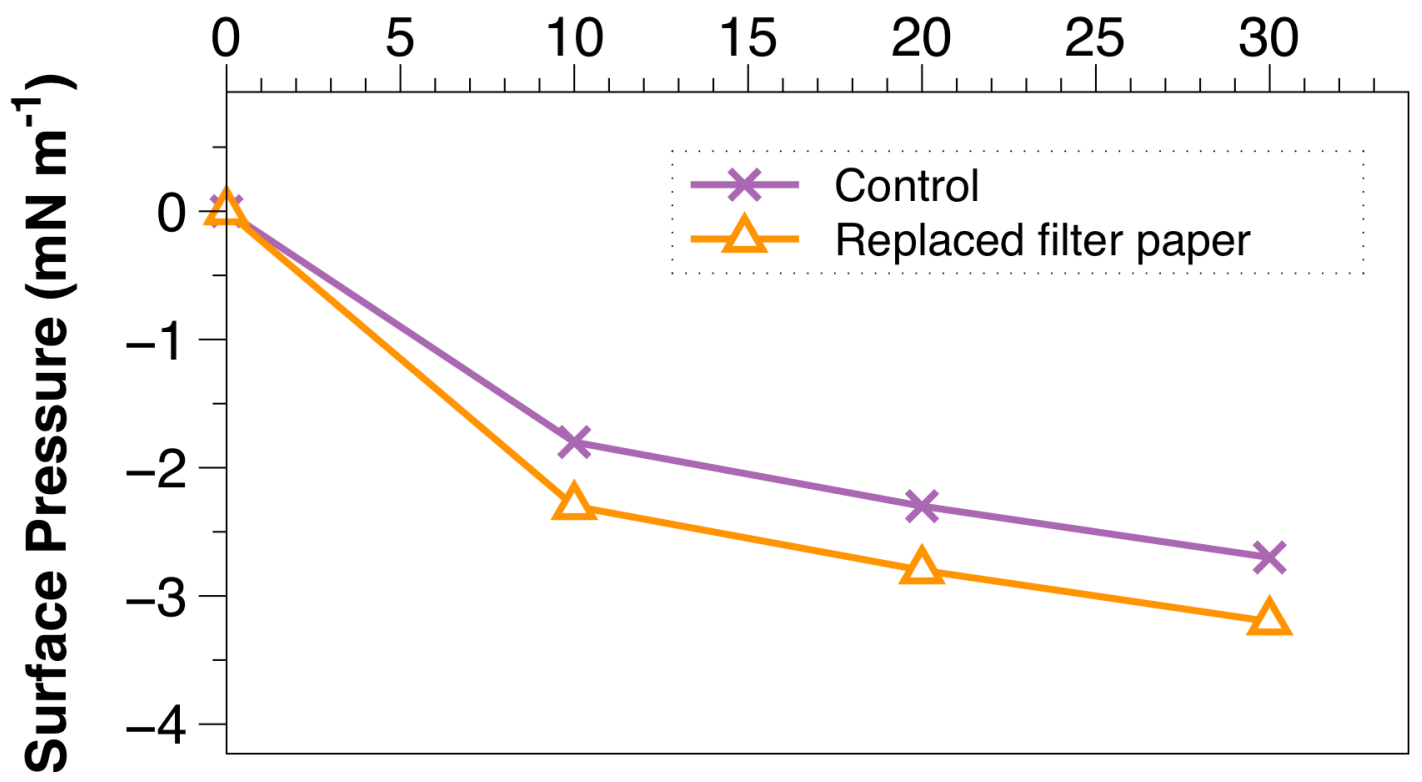


Time (minutes)
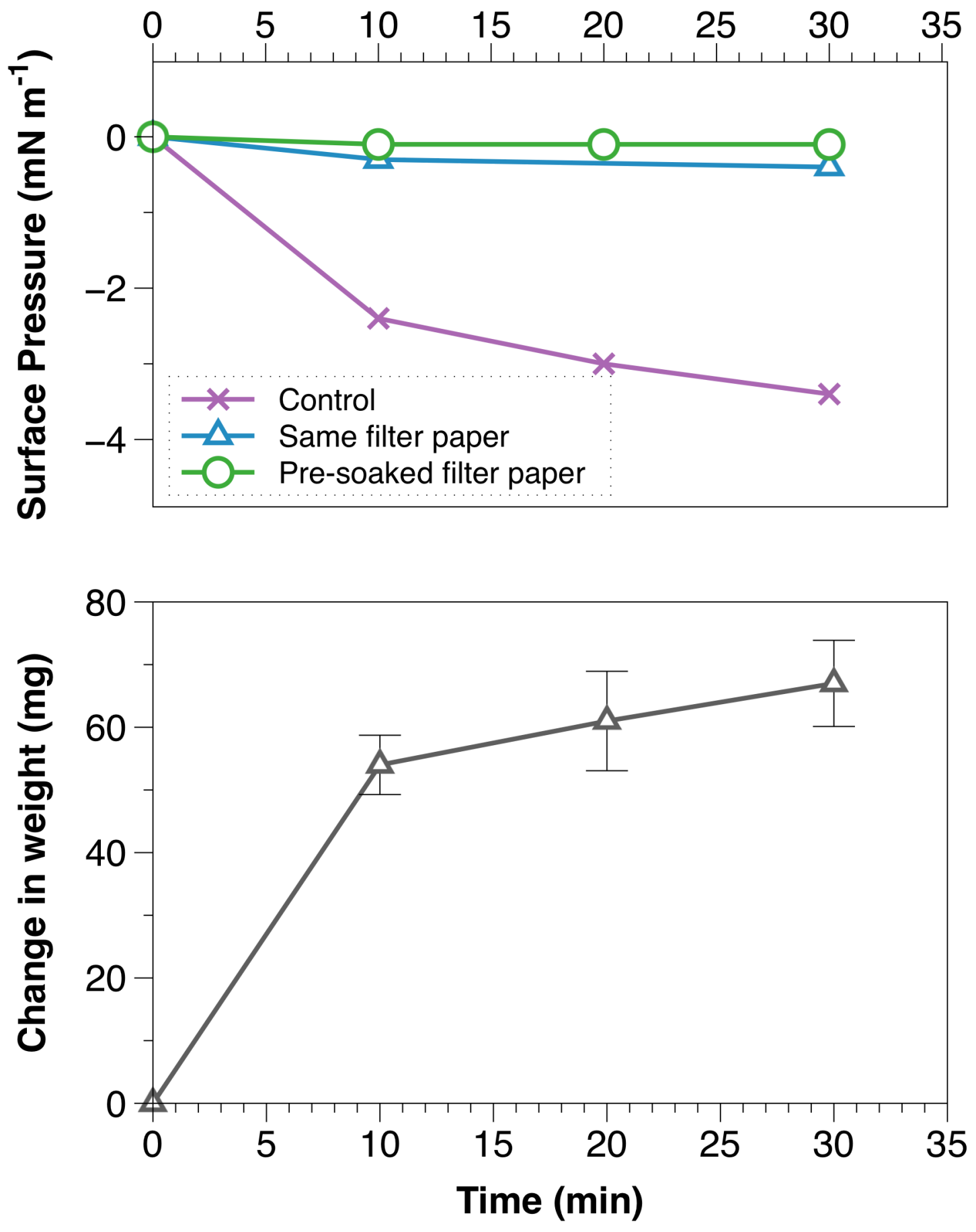

Figure a. Schematic of the forces acting on a Wilhelmy plate, illustrating the parameters required for the determination of surface pressure.

Figure b. The results of three consecutive experiments in which the surface pressure of water in a Langmuir Blodgett trough was recorded over time (from trough setup) using a filter paper Wilhelmy plate. (Raw data available at university repository)

Figure c. Changes in the measured surface pressure, with $\mathrm{X}$ and $\Delta$ representing data from two different filter paper Wilhelmy plates, recorded sequentially without changing the water in the trough. (Raw data available at university repository)

Figure d. Plot of the surface pressure measured using a Wilhelmy balance with a filter paper Wilhelmy plate. X represents the data from the first plate, $\mathrm{O}$ the data from the same filter paper plate, but with the water in the LB trough changed, while $\Delta$ represents the data obtained after changing the water in the trough and replacing the plate by a new 
filter paper plate that was pre-soaked in deionised $\mathrm{H}_{2} \mathrm{O}$ for 2 hours. (Raw data available at university repository)

Figure e. Change in the average weight of three filter papers, measured using an Ohaus GA20oD balance. The error bars represent the standard deviation of the three measurements at each time point. (Raw data available at university repository) 


\section{Results \& Discussion}

The initial study focused on quantification of the drift in the readings taken with the Wilhelmy plate method. This was critical to understand the problem and its effect on monolayer characterisation. For the initial characterisation, the trough was filled with distilled water and a new piece of filter paper was hung on the Wilhelmy balance. Once the water had visibly saturated the filter paper, the sensor was 'zeroed'- this data point was used as $t=0$. The surface pressure was then recorded over a 3 hours period. The experiment was repeated three times, on three consecutive days. The data collected during the three experiments are shown in Figure $b$.

Figure $b$ shows that the form of the drift is repeatable, with the measured surface pressure taking approximately around $150 \mathrm{~min}$ to reach equilibrium. There is a clear variation between each run, with the average drift equating to approximately $10 \%\left(3-4 \mathrm{mN} \mathrm{m}^{-1}\right)$ of the surface pressure that would be typically used during transfer of a monolayer onto a substrate during Langmuir-Blodgett deposition.

As the drift was observed to approach a stable plateau, it was assumed that this problem was caused by one or more parts of the system requiring time to equilibrate. Given that the system is comprised of water and a sensor, this limits the number of possible causes. The two key hypotheses considered were the presence of contamination at the liquid surface that causes the surface pressure to take time to equilibrate, and instabilities in the Wilhelmy balance mechanism. Firstly, the possible contamination of the liquid surface was considered. A control experiment was undertaken to ensure that the measured surface pressure had reached equilibrium. The filter paper was then removed and replaced with a new piece. This replacement of the filter paper was undertaken carefully to minimize disturbance of the water's surface. Once fully wetted, the Wilhelmy sensor was again 'zeroed' and the resulting drift in the surface pressure readings were recorded. The data collected are shown in Figure $c$.

Figure $c$ shows little difference in the magnitude of the drift in the surface pressure readings between the two experiments. If the water (or contamination thereof) was the cause of the drift then with the first plate we might expect to see a slow change in the surface pressure as the interface layer forming contaminate reached an equilibrium. However, once formed this layer should not be significantly disrupted by the addition of a new plate as it has been already reached in equilibrium (typified by a decrease in the rate of change in surface pressure). However in Figure $c$, the drift with the first and second plates are similar in magnitude, which indicates that the change is not due to a layer forming from the contaminant in the sub-phase. This indicates that the drift is a property of the Wilhelmy sensor system and not of the water.

Figure $d$ shows a reduction in the magnitude of the drift of the Wilhelmy surface pressure, when the filter paper plate had been pre-soaked in water. As discussed, the Wilhelmy plate measures the weight of the plate to determine the surface pressure of the water. The drift observed after the filter paper Wilhelmy plate has been wetted, suggests the filter paper is increasing in the weight. It has been suggested that, the reason for this effect is, although appearing fully wetted, the filter paper continues to absorb more water when immersed in the trough, increasing the overall weight, which has recorded by the pressure sensor.

To test the swelling theory, the change in weight of the filter paper was measured using an Ohaus GAzooD balance (+/$0.2 \mathrm{mg}$ precision). Three filter paper plates were wetted in a small vial of distilled water and weighed immediately. Once weighed, the plates were returned of the vial of distilled water, where they were allowed to soak for 30 min. During this period, they were weighed at the intervals to record any change in the wetted weight. The results are shown in Figure e.

Figure e shows that the plates gain weight at a similar rate to the drift observed in the measurement of surface pressure by the Wilhelmy balance, indicating that the surface pressure measurement drift is the result of the filter paper plates slowly gaining weight.

\section{Conclusions}

The use of dry filter paper causes a drift in the readings taken from a Wilhelmy surface pressure sensor for up to 2 hours after initial setup. The slow increase in the water holding capacity of the filter paper has an influence on the weight of the plate and subsequently the measurements obtained from the Wilhelmy balance. The soaking of filter paper in water prior to use as a Wilhelmy plate reduces the time taken for the surface pressure measurements to stabilise.

\section{Limitations}

In this study, we have been limited to a single brand of plate paper. While this is the brand typically sold by KSV Nima, one the biggest users of filter paper based Wilhelmy plates, further studies using other filter paper materials would be useful to researchers using these systems. 


\section{Additional Information}

Methods and Supplementary Material

Please see https://sciencematters.io/articles/201611000016.

\section{Funding Statement}

This work was supported by the Engineering and Physical Sciences Research Council (EPSRC), UK grant EP/Ho2252X.

Ethics Statement

Not Applicable.

\section{Citations}

[1] Ludwig Wilhelmy. "Ueber die Abhängigkeit der CapillaritätsConstanten des Alkohols von Substanz und Gestalt des benetzten festen Körpers". In: Annalen der Physik und Chemie 195.6 (1863), pp. 177-217. Dor: 10 . 1002 / andp . 18631950602 uRL: https : / / doi . org / 10 . 1002 \% 2Fandp . 18631950602

[2] M.C. Petty. "Langmuir-blodgett films". In: Endeavour 7.2 (Jan. 1983), pp. 65-69. DOI: 10 . 1016/s0160-9327 (83) 80004-0 URL: https : //doi.org/10.1016\%2Fs0160-9327\%2883\% 2980004-0
[3] G L Gaines. "Insoluble Monolayer at Liquid-Gas Interfaces". In: fohn Wiley and Sons (1966).

[4] Faridah L. Supian et al. "Interaction between Langmuir and Langmuir-Blodgett Films of Two Calix[4]arenes with Aqueous Copper and Lithium Ions". In: Langmuir 26.13 (July 2010), pp. 10906-10912. DOI: 10 . 1021/la100808r URL: https : //doi.org/10.1021\%2Fla100808r

[5] G. S. Lapham, D. R. Dowling, and W. W. Schultz. "In situ forcebalance tensiometry". In: Experiments in Fluids 27.2 (July 1999), pp. 157-166. DOI: 10 . 1007/s003480050340 URL: https : //doi.org/10.1007\%2Fs003480050340. 
2017-01-12

\section{A solution to the slow stabilisation of surface pressure sensors based on the Wilhelmy method}

\section{Partridge, Matthew}

\section{Sciencematters AG}

Matthew Partridge, Frank Davis, Stephen W. James, Seamus P. Higson, Ralph P. Tatam, A solution to the slow stabilisation of surface pressure sensors based on the Wilhelmy method, Matters: stories can wait. science can't, 2017. DOI: 10.19185/matters.201611000016

https://dspace.lib.cranfield.ac.uk/handle/1826/11460

Downloaded from Cranfield Library Services E-Repository 